\title{
Nonstandard Roughness of Terraced Surfaces
}

\author{
Stefan Gheorghiu ${ }^{1}$ and Peter Pfeifer ${ }^{1,2}$ \\ ${ }^{1}$ Physics Department, University of Missouri, Columbia, Missouri 65211 \\ ${ }^{2}$ Institute for Physical Sciences, Inc., Los Alamos, New Mexico 87544
}

(Received 23 December 1999)

\begin{abstract}
We present a class of surfaces which are simultaneously flat and rough over the same range of lengths. They are self-affine, have well-defined roughness exponents, and consist of terraces of all sizes. The coexistence of flat and rough makes them respond to different external interactions with variable roughness. We demonstrate this for optical scattering (including $\mathrm{x}$ rays), two wetting situations, diffusion currents, and catalysis. A terraced $\mathrm{Cu}$ surface is a "self-assembled" experimental example, and designs for nanoand micromachined examples are presented.
\end{abstract}

PACS numbers: 68.35.Ct, 61.10.Kw, 61.43.Hv, 68.10.Jy

Terraced surfaces, as produced by molecular beam epitaxy [1,2], etching [3], electrodeposition [4], and other surface growth/removal processes, are the ultimate laboratory for studying how rough surfaces form and interact with other systems. They are locally flat [5], rough by virtue of the steps between terraces, and often have well-defined roughness exponents $H_{q}$ (self-affinity),

$$
C_{q}(\vec{l}):=\left[\left\langle|h(\vec{x}+\vec{l})-h(\vec{x})|^{q}\right\rangle_{\vec{x}}\right]^{1 / q} \sim b(|\vec{l}| / b)^{H_{q}}
$$

$(q>0)$ [6]. The reduction of the average height difference between sites $\vec{x}$ and $\vec{x}+\vec{l}$ to two parameters $-H_{q}$ and the length $b$-is a strong form of a complex system obeying a simple law. The $H_{q}$ 's were introduced [7] to study self-affine surfaces whose scaling properties differ from point to point, called multiaffine or turbulent. But remarkably little progress has been made in understanding how they control functional properties (structure-function relations). We present such a study, focusing on that different properties may be governed by different orders $q$ in $H_{q}$, i.e., different statistics of the height differences. In parallel, we introduce a class of surfaces for which $H_{q}$ varies strongly, from $\infty$ to less than 1 , as $q$ varies from 0 to $\infty$. These surfaces will be particularly sensitive to the selection of different $q$ 's by different functions.

The difference between surfaces with weakly varying $H_{q}$ (over a finite interval), which we call standard rough, and the new class with strongly varying $H_{q}$, which we call nonstandard rough, is noteworthy. In standard roughness, terraces have nearly the same size, and the power law (1) resides at scales $|\vec{l}|$ above the largest terrace size. The flat and rough regime are separated: the surface is flat below the smallest terrace size and rough above the largest terrace size. A nonstandard rough surface, in contrast, has terraces of all sizes, and the power law occurs at scales smaller than the largest terrace size. The flat and rough regime are intertwined: the surface is simultaneously flat and rough over the same range of lengths; it is flat across any single terrace and rough across the adjacent family of terraces spanning the same length. The coexistence of flat and rough enables the surface to behave as flat or rough ("switchable roughness"), depending on whether it acts as optical scatterer, adsorbent, substrate for liquid droplets, receptor of a diffusion current, or catalyst, as we shall show.

An experimental example which meets all prerequisites for nonstandard roughness, grown by Zuo and Wendelken [2], is shown in Fig. 1. From the data for $C_{2}(\vec{l})$ [8], we find $H_{2}=0.84 \pm 0.02$ and $b \sim 9 \times 10^{-6} \AA$ for $4 \AA<|\vec{l}|<90 \AA$. It has terraces of all sizes; the average terrace size is $43 \AA$, and the largest terrace size is $110 \AA$. Zuo and Wendelken interpreted their $H_{2}$ as consistent with the value $H_{2}=1$ expected for mound growth [2]. We offer the interpretation that the exponent is equally consistent with nonstandard roughness, by presenting a model which is monotone (just as the uphill part of a mound is), has $H_{2}<1$ over an unlimited range of scales (which rules out the scenario that $H_{2}<1$ can occur only as a crossover), and represents mound growth by $H_{1}=1$ instead of $H_{2}=1$ [9].

The model is the triadic Cantor staircase (Fig. 2c), the archetype of a surface with terraces of all sizes. To explore scaling below the largest terrace size, we computed $C_{q}(l)$ numerically and analytically. Positions $x$ and heights

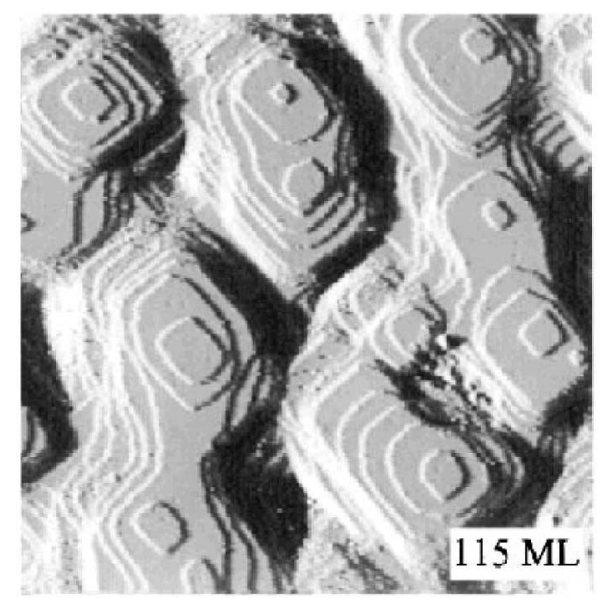

FIG. 1. Terraced surface of 115 monolayers of $\mathrm{Cu}$ on $\mathrm{Cu}(100)$ with area $1000 \times 1000 \AA^{2}$ (from [2] with permission). 


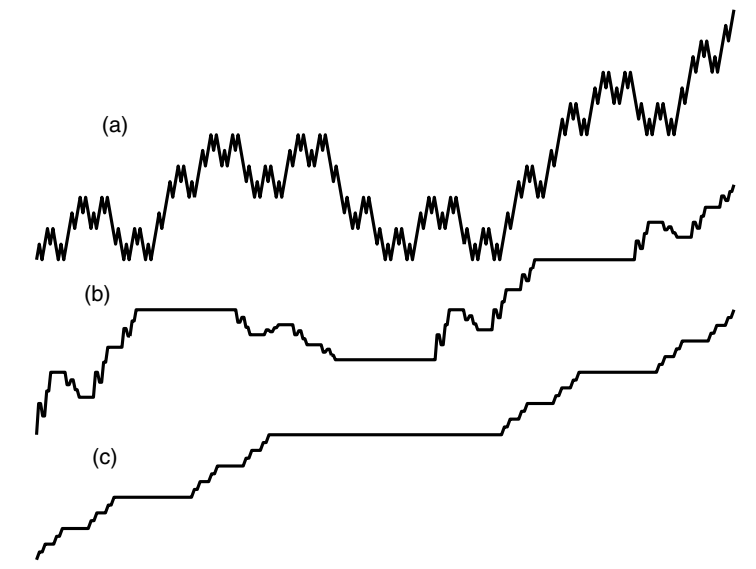

FIG. 2. Standard (a) and nonstandard (b),(c) rough surfaces from (3). (a) $p_{1}=-p_{2}=p_{3}=p_{4}=1 / 2 ; \quad H_{1}=1 / 2$. (b) $p_{2}=p_{4}=p_{6}=0, \quad p_{1}=0.5, \quad p_{3}=-0.2, \quad p_{5}=0.4$, $p_{7}=0.3 ; H_{1}=0.83$. (c) $p_{2}=0, p_{1}=p_{3}=1 / 2 ; H_{1}=1$.

$h(x)$ vary from 0 to 1 , the largest terrace size is $1 / 3$, and $C_{q}(l)=\left[(1-l)^{-1} \int_{0}^{1-l}|h(x+l)-h(x)|^{q} d x\right]^{1 / q}(0<$ $l<1)$. The numerical results satisfy (1) over as many decades of $l$ as desired, with $H_{1}=1.00, H_{2}=0.81$, and $H_{3}=0.75$ (Fig. 3). The analytic result is [10]

$$
\begin{aligned}
& C_{q}\left(3^{-j}\right)=\left[\frac{2^{j+1}-2}{1-3^{-j}} \sum_{i=j+1}^{\infty} 3^{-i} 2^{-i q} \sum_{\substack{k=1 \\
k \text { odd }}}^{2^{i-j}-1} k^{q}\right]^{1 / q} \\
& C_{1}\left(3^{-j}\right)=\frac{1-2^{-j}}{3^{j}-1} \sim 3^{-j}, \\
& C_{2}\left(3^{-j}\right)=\left[\frac{3}{5} \frac{2^{-j}\left(1-2^{-j}\right)}{3^{j}-1}\right]^{1 / 2} \sim \sqrt{\frac{3}{5}} 3^{-j(\ln 6 / \ln 9)}
\end{aligned}
$$

$(j=1,2, \ldots)$, whence $H_{1}=1$ and $H_{2}=\ln 6 / \ln 9=$ 0.81 . Further evaluation of (2) gives $H_{3}=\ln 12 / \ln 27=$ 0.75 with prefactor $(4 / 5)^{1 / 3}$, and $H_{q}=q^{-1}+(1-$ $\left.q^{-1}\right) \ln 2 / \ln 3$ for general $q$. Equation (2) includes all prefactors and finite-size corrections to scaling, and to our knowledge is the first exact height-difference correlation function of a self-affine surface. Thus the Cantor staircase has well-defined roughness exponents, the exponents vary from $\infty$ to 0.63 , and the scaling regime lies below the largest terrace size $(l \leq 1 / 3)$.

The existence of roughness exponents for the staircase is unexpected [11] and motivates the term "nonstandard rough." $H_{q}$ diverges at $q=0$ because the staircase is flat almost everywhere in the continuum limit (infinitesimal step height): as $q \rightarrow 0$, the mean (1) approaches the geometric mean, which is zero whenever $h(x+l)-h(x)=$ 0 in some interval of $x$, whence $H_{0}=\infty$. The strong variation of $H_{q}$ is due to strongly varying height differences: for given $l$, it is not true that $|h(x+l)-h(x)| \sim b(l / b)^{H}$ for most $x$ and some $H$, as (1) may suggest; instead the histogram of height differences is hierarchically peaked, with an isolated peak at 0 , far from Gaussian. In contrast, the surfaces with which roughness exponents have

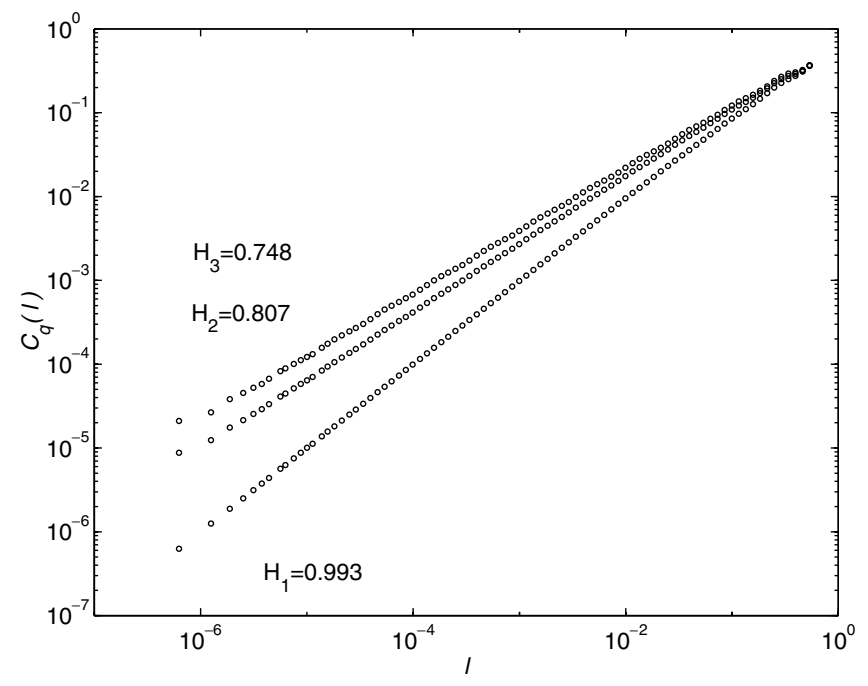

FIG. 3. Height-difference correlation function for $q=1,2,3$ of the Cantor staircase.

been associated previously are nowhere differentiable in the continuum limit, typically by fluctuating at all scales (Fig. 2a). In the simplest case, the height-difference histogram is Gaussian, with $q$-independent $H_{q}$ (monoaffine surface); at most, $H_{q}$ varies over a finite interval because without flat segments in the continuum limit, $H_{0}$ is finite and there is no room for scaling below the largest terrace size. In the continuum limit, a standard rough surface is nowhere differentiable, while a nonstandard rough surface has zero derivative almost everywhere.

Flatness almost everywhere offers the prospect of fabricating nonstandard rough surfaces by nano- and micromachining, including high aspect-ratio etching [12]. A profile with positions $0 \leq x \leq L_{\|}$, heights $0 \leq h(x) \leq L_{\perp}$, and prescribed $H_{q}$ 's can be constructed as follows. We divide the $x$ interval into $m$ equal parts and distribute its "mass," set equal to $L_{\perp}$, by assigning the mass fraction $p_{i}$ (positive, zero, or negative) to the $i$ th part, where $\left|p_{i}\right| \leq 1$ and $\sum_{i=1}^{m} p_{i}=1$. Recursive mass distribution generates a self-similar measure $\mu$. The cumulative distribution function, $h(x):=\int_{0}^{x} d \mu\left(x^{\prime}\right)$, is our profile. If $p_{i} \geq 0$ for all $i, \mu$ is a positive measure and the profile is nondecreasing. If $p_{i}<0$ for some $i, \mu$ is a signed measure and the profile fluctuates at all (Fig. 2a) or some (Fig. 2b) scales. If $p_{i}=0$ for some $i, \mu$ lives on a Cantor set and the profile consists of terraces of all sizes (Figs. 2b and 2c). Standard rough profiles are those with $\prod_{i=1}^{m} p_{i} \neq 0$, and nonstandard rough profiles are those with $\prod_{i=1}^{m} p_{i}=0$. In either case, the profile is the fixed point of the union of $m$ affinities and thus self-affine. The roughness exponents, length $b$, and smallest terrace size, $l_{0}$, are

$$
\begin{gathered}
H_{q}=\ln \left(\left[m^{-1} \sum_{i=1}^{m}\left|p_{i}\right|^{q}\right]^{1 / q}\right) / \ln \left(m^{-1}\right), \\
b=L_{\|}\left(L_{\perp} / L_{\|}\right)^{1 /\left(1-H_{\infty}\right)} \\
l_{0}=m^{- \text {(number of iterations) }} L_{\|},
\end{gathered}
$$


with $H_{q} \leq 1$ if $q \geq 1[10,13]$. This blueprint for machined self-affinity highlights the hallmark of nonstandard and standard roughness: after etching, the largest terrace size is $L_{\|} / m$ and $l_{0}$, respectively.

The interaction of a self-affine surface with an object of size $l$ depends on whether $C_{q}(l) / l \gg 1$ [steep regime, $(l-b)\left(H_{q}-1\right)>0$ ] or $C_{q}(l) / l \ll 1$ [shallow regime, $\left.(l-b)\left(H_{q}-1\right)<0\right]$. The roughness increases or decreases if $(l-b)\left(H_{q}-1\right)$ does so. This compares the roughness of a surface at two different scales, or of two different surfaces at the same scale. For example, the Cantor staircase is minimally rough in the steep regime, $l \ll b$, and maximally rough in the shallow regime, $l \gg b$, by $H_{1}=1$.
The interactions we have selected are optical scattering (differential cross section $d \sigma / d \Omega$ vs wave-vector transfer $\vec{q}_{\|}$and $q_{\perp}$ parallel and perpendicular to the basal plane $S_{0}$ ), adsorption of a liquid film (film volume $V$ vs vapor pressure $P$ approaching the coexistence pressure $P_{0}$ of bulk liquid and gas), a liquid droplet of radius $R$ (contact angle $\theta$ vs contact angle $\theta_{0}$ on a flat surface), mass or heat transfer across the surface (flux $\Phi$ vs diffusivity $D$ and permeability $W$ ), and catalysis at terrace edges (flux $\Phi$ vs catalyst size $L_{\|}$). We take the surface as the Cartesian product of the $L_{\|} \times L_{\perp}$ profile and the interval $\left[0, L_{\|}\right]$, and approximate crossovers between different power laws as sharp. Our results are [10]

$$
\begin{aligned}
\frac{d \sigma}{d \Omega} & =\pi^{2}\left(1-n^{2}\right)^{2} \lambda^{-4} q_{\perp}^{-2} \int_{S_{0}} d^{2} r e^{i \vec{q} \| \cdot \vec{r}} \int_{S_{0}} d^{2} x e^{i q_{\perp}[h(\vec{x}+\vec{r})-h(\vec{x})]} \\
& =\pi^{2}\left(1-n^{2}\right)^{2} \lambda^{-4} L_{\|}^{2} q_{\perp}^{-2} \int_{S_{0}} d^{2} r e^{i \overrightarrow{q_{\|}} \cdot \vec{r}} \sum_{j=0}^{\infty} \frac{\left(i q_{\perp}\right)^{2 j}}{(2 j) !}\left[C_{2 j}(\vec{r})\right]^{2 j} \\
& \sim \pi^{2}\left(1-n^{2}\right)^{2} \lambda^{-4} L_{\|}^{2} q_{\perp}^{-2} \int_{S_{0}} d^{2} r \exp \left\{i \overrightarrow{q_{\|}} \cdot \vec{r}-q_{\perp}^{2}\left[C_{2}(\vec{r})\right]^{2} / 2\right\} \\
& \sim \frac{\pi^{3}}{2}\left(1-n^{2}\right)^{2} L_{\|}^{2}(b / \lambda)^{4} \Gamma\left(1+1 / H_{2}\right) \min \left\{\left(\frac{L_{\|}}{b}\right)^{2+2 H_{2}},\left|\frac{b q_{\perp}}{\sqrt{2}}\right|^{-2-2 / H_{2}}, \frac{\sin \left(\pi H_{2}\right) \Gamma^{2}\left(1+H_{2}\right)}{\pi \Gamma\left(1+1 / H_{2}\right)}\left|\frac{b \vec{q}_{\|}}{2}\right|^{-2-2 H_{2}}\right\},
\end{aligned}
$$

$$
\begin{gathered}
V=L_{\|}^{2} \begin{cases}{\left[-\alpha /\left(k_{\mathrm{B}} T \ln \left(P / P_{0}\right)\right)\right]^{1 / 3}} & \text { for } 0 \leq H_{\infty}<1 / 2, \\
(b / 6)\left[-\gamma /\left(b \rho k_{\mathrm{B}} T \ln \left(P / P_{0}\right)\right)\right]^{H_{\infty} /\left(2-H_{\infty}\right)} & \text { for } 1 / 2<H_{\infty} \leq 1,\end{cases} \\
\cos \theta= \begin{cases}\min \left\{1,\left(b / l_{0}\right)^{1-H_{1}} \cos \theta_{0}\right\} & \text { for } 0<\theta_{0}<\pi / 2, l_{0} \ll \min \{b, R\}, \\
\max \left\{-1,\left(b / l_{0}\right)^{1-H_{1}} \cos \theta_{0}\right\} & \text { for } \pi / 2<\theta_{0}<\pi, l_{0} \ll \min \{b, R\}, \\
\min \left\{1,\left[1+\frac{1}{2}\left(b / l_{0}\right)^{2-2 H_{2}}\right] \cos \theta_{0}\right\} & \text { for } 0<\theta_{0}<\pi / 2, b \ll l_{0} \ll R, \\
\max \left\{-1,\left[1+\frac{1}{2}\left(b / l_{0}\right)^{2-2 H_{2}}\right] \cos \theta_{0}\right\} & \text { for } \pi / 2<\theta_{0}<\pi, b \ll l_{0} \ll R,\end{cases} \\
\Phi=W c_{0} L_{\|}^{2} \begin{cases}1 & \text { for } D / W<b\left(b / l_{0}\right)^{-H_{1}}, \\
{\left[\left(b / l_{0}\right)^{H_{1}} D /(W b)\right]^{1-H_{1}}} & \text { for } b\left(b / l_{0}\right)^{-H_{1}}<D / W<b\left(b / l_{0}\right)^{1-H_{1}}, \\
\left(b / l_{0}\right)^{1-H_{1}} & \text { for } b\left(b / l_{0}\right)^{1-H_{1}}<D / W,\end{cases}
\end{gathered}
$$

$$
\Phi=W c_{0} a_{0} l_{0}\left(L_{\|} / l_{0}\right)^{2-\lim _{q \rightarrow 0} q H_{q}} .
$$

Result (6d) is the first complete expression for power-law scattering from a self-affine surface (wavelength $\lambda$ in the steep or shallow regime, index of refraction $n$, Born approximation). The three terms are the central peak (finite-size cutoff at small $\vec{q}_{\|}, q_{\perp}$ ), diffuse scattering at the specular condition $\left(\vec{q}_{\|}=0\right)$, and off-specular scattering (independent of $q_{\perp}$ at large $\vec{q}_{\|}$). The specular power law is due to Sinha et al. [14] and has become a mainstay in x-ray and neutron scattering; the off-specular power law is new. Together they interpolate in a remarkable way between a marginally flat $\left(H_{2}=0\right)$ and a marginally self-similar $\left(H_{2}=1\right)$ surface: $(6 \mathrm{c})$ at $H_{2}=0,1$ without cutoff is proportional to $q_{\perp}^{-2} \exp \left(-q_{\perp}^{2} b^{2} / 2\right) \delta\left(\vec{q}_{\|}\right)$(specular reflection, Debye-Waller factor for rms roughness $b$ ) and $q_{\perp}^{-4} \exp \left(-\left|\vec{q}_{\|} / q_{\perp}\right|^{2} / 2\right.$ ) (Porod law at the specular condition), so the off-specular intensity falls off faster than any power of $\left|\vec{q}_{\|}\right|$. The off-specular prefactor in (6d) vanishes at $H_{2}=0,1$ accordingly. In between, the intensity falls off rapidly (slowly) with increasing vertical (parallel) momentum transfer. It follows that, for fixed $\vec{q}_{\|}, q_{\perp}$, the intensity peaks at an $\mathrm{H}_{2}$ value between 0 and 1 (e.g., at $H_{2}=0.74$ if $\left|b \vec{q}_{\|}\right|=\left|b q_{\perp}\right|=1$ ). Sinha et al. obtained the specular power law for a Gaussian height-difference distribution $\left(H_{q}\right.$ independent of $\left.q\right)$. We make no such assumption: under weak conditions, (6a) gives (6b); (6b) shows that scattering from a self-affine surface involves $\mathrm{H}_{2}, \mathrm{H}_{4}, \ldots$; and (6c), the analog of Guinier's approximation in scattering from polymers, identifies $\mathrm{H}_{2}$ as the leading exponent. The domain of (6d) is $\left|l_{0} \vec{q}_{\|}\right| \ll 1$.

In (7) and (8) we answer the question [15] whether roughness enhances wetting, and establish two phase 
transitions in which $H_{q}$ plays the role of temperature. The adsorption isotherm (7) does so for complete wetting in the shallow regime, $b^{3} \rho \ll 1[16]$. The film has number density $\rho$ and is in equilibrium with its vapor at temperature $T$. The substrate potential $-\alpha /{\text { (distance })^{3}}^{3}$ favors the liquid-gas interface at distance equal to that on a flat surface; the liquid-gas surface tension $\gamma$ seeks to fill the valleys with liquid. At low roughness, $H_{\infty}<1 / 2$, the film grows with $P$ as on a flat surface: wetting is not enhanced. At $H_{\infty}>1 / 2$, capillary instability sets in and the film grows faster: wetting is enhanced. The film is controlled by $H_{\infty}$ because the meniscus across a valley is always anchored at the highest mountain peaks $[q \rightarrow \infty$ in (1)]. For partial wetting, (8), roughness alters the contact angle, according to Wenzel's law [15]. The surface area in Wenzel's law brings in $H_{1}$ and $H_{2}$. In the steep regime, $l_{0} \ll b$, enhanced wetting $\left(0 \leq \theta<\theta_{0}<\pi / 2\right)$ or drying $\left(\pi / 2<\theta_{0}<\theta \leq \pi\right)$ are strong and controlled by $H_{1}$. The drop completely wets or dries the surface at small $H_{1}$ and $l_{0} / b(\cos \theta= \pm 1$, wetting or drying transition). In the shallow regime, $b \ll l_{0}$, enhanced wetting or drying are weak and controlled by $\mathrm{H}_{2}$.

The flux (9) is the integrated diffusion current across the surface for the reaction-diffusion problem $\nabla^{2} c=0$, $c=c_{0}$ at the source, $D \nabla c \cdot($ surface normal $)=W c$ at the surface, where $c$ is concentration and $l_{0} \ll b \ll L_{\|}$. We take the surface as membrane, but with obvious reinterpretations $\Phi$ may also be a chemical vapor deposition rate, catalytic reaction rate, heat flux, or electric current across an electrode [17]. The power laws describe complete screening ( $W$ large - random walkers react at tips), partial screening ( $W$ intermediate - walkers enter fjords before reacting), and no screening ( $W$ small - walkers visit the entire surface before reacting). They parallel the screening regimes for self-similar surfaces [18]. The nonlinear flux suggests interesting high-performance applications. For example, the biomimetic nonstandard rough surface, (3)-(5) with $m=13$ and $p_{i}=\cos (i \pi / 2)$ ("self-affine gill"), has $\Phi \propto W^{H_{1}}$ with $H_{1}=0.24$ for partial screening. The weak dependence on $W$ makes the membrane or catalyst error tolerant (small flux loss upon loss of permeability, small activity loss upon self-poisoning).

The flux (10) is a variant of (9) for an unscreened catalyst. Reaction occurs at terrace edges, so the active surface area is (reactant diameter $\left.a_{0}\right) \times$ (total edge length), with $a_{0}<l_{0}$. The number of terraces of length $\geq l$ grows with decreasing $l$ as $l^{\lim _{q \rightarrow 0} q H_{q}-1}$ on a nonstandard rough surface. Thus (10) reflects that the limit $q \rightarrow 0$ zooms in on vanishing height differences.

To summarize, structural highlights of the new surfaces are hierarchical height-difference distributions, flatness almost everywhere, scaling below the largest terrace size, and strongly varying $H_{q}$ 's. Functional highlights are the switchable response to external interactions (different interactions sample different $q$ 's, illustrated for five examples), switchable response by structural and dynamic thresholds (steep/shallow regime, "phase transitions"), and macroscopic amplification of microscopic lengths $\left(b, l_{0}\right)$. We believe this creates a novel library for the design of multifunctional surfaces.

We thank J.-K. Zuo, P. Miceli, F. Family, B. Sapoval, and M.-O. Coppens for valuable discussions, and the Petroleum Research Fund for support.

[1] J. Vrijmoeth et al., Phys. Rev. Lett. 72, 3843 (1994).

[2] J.-K. Zuo and J.F. Wendelken, Phys. Rev. Lett. 78, 2791 (1997).

[3] J. J. Boland and J.H. Weaver, Phys. Today 51, No. 8, 34 (1998).

[4] K. Sieradzki, S. R. Brankovic, and N. Dimitrov, Science 284, 138 (1999).

[5] Here flat means $h(\vec{x})-h(\vec{y})=0$ for all relevant $\vec{x}, \vec{y}-$ not $\lim _{|\vec{l}| \rightarrow \infty} C_{2}(\vec{l})<\infty$ as for a crystal facet $[\mathrm{H}$. van Beijeren and I. Nolden, in Structure and Dynamics of Surfaces II, edited by W. Schommer and P. von Blanckenhagen (Springer, Berlin, 1987), p. 259].

[6] (a) F. Family and T. Vicsek, Dynamics of Fractal Surfaces (World Scientific, Singapore, 1991); (b) A.-L. Barabási and H. E. Stanley, Fractal Concepts in Surface Growth (Cambridge University Press, Cambridge, 1995); (c) P. Meakin, Fractals, Scaling, and Growth Far from Equilibrium (Cambridge University Press, Cambridge, 1998).

[7] A.-L. Barabási and T. Vicsek, Phys. Rev. A 44, 2730 (1991).

[8] J.-K. Zuo (private communication). No other functions $C_{q}$ or heights $h(\vec{x})$ were recorded.

[9] Another likely example is the equilibrium shape of a crystal at absolute zero. Its facets are separated by edges forming a Cantor set [S. E. Burkov, J. Phys. (Paris) 46, 317 (1985)]. We expect $H_{2}<1$ across facets of different sizes.

[10] S. Gheorghiu and P. Pfeifer (to be published).

[11] Meakin, Fractals, Scaling, and Growth Far from Equilibrium [Ref. [6(c)]], p. 82.

[12] K. Wilder et al., Appl. Phys. Lett. 73, 2527 (1998); F. S. S. Chien et al., Appl. Phys. Lett. 75, 2429 (1999); F. Yongqi et al., Rev. Sci. Instrum. 71, 1006 (2000).

[13] We define $b$ by $\max _{x}|h(x+b)-h(x)|=b$. For $p_{i} \neq$ 0, Eq. (3) was obtained in [7]. The fractal dimension (box counting at decreasing scales) of the profiles equals $2-H_{1}$ [10] [see also S. Jaffard, SIAM J. Math. Anal. 28, 944 (1997)]. Thus the profile in Fig. 2b, flat almost everywhere, has infinite length. If $\sum_{i=1}^{m} p_{i} \neq 1$, the heights $h(x)$ converge to 0 if $\left|\sum_{i=1}^{m} p_{i}\right|<1$, and do not converge else.

[14] S. K. Sinha et al., Phys. Rev. B 38, 2297 (1988).

[15] C. Borgs et al., Phys. Rev. Lett. 74, 2292 (1995).

[16] P. Pfeifer and K.-Y. Liu, in Surface Disordering: Growth, Roughening, and Phase Transitions, edited by R. Jullien et al. (Nova Science, New York, 1992), p. 295; P. Pfeifer and K.-Y. Liu, Stud. Surf. Sci. Catal. 104, 625 (1997).

[17] M. Filoche and B. Sapoval, Phys. Rev. Lett. 84, 5776 (2000).

[18] P. Pfeifer and P.J. Hagerty, in Fractals and Chaos in Chemical Engineering, edited by M. Giona and G. Biardi (World Scientific, Singapore, 1997), p. 151. 